\title{
The Self-Assembling Growth of Copper Nanowires for
}

\section{Transparent Electrodes}

\author{
Wu Junqing, ${ }^{1}$ Guo Le, ${ }^{1}$ Wen Min, ${ }^{1}$ Bu Tongle, ${ }^{1}$ Zhou Peng, ${ }^{1}$ Zhong Jie, ${ }^{1}$ Huang Fuzhi, ${ }^{1}$ and \\ Zhang Qi, ${ }^{1,2}$
}

(1.School of Materials Science and Engineering, and State Key Laboratory of Advanced Technology for Materials Synthesis and Processing, Wuhan University of Technology, Wuhan 430070, China;

(2.School of Aerospace, Transport and manufacturing, Cranfield University, Cranfield, Bedfordshire, MK43 0AL, UK)

\begin{abstract}
Cu}$ nanowires (CuNWs) have attracted great interest due to their growing application in transparent electrodes. In this work, we have prepared long $(15-40 \mu \mathrm{m})$, thin (diameter of $20 \pm 5 \mathrm{~nm}$ ), well-dispersed CuNWs. The high-resolution TEM and selected area electron diffraction showed the CuNWs were single crystalline. To investigate the growth mechanism,we examined the microstructure of the samples at different reaction times. It was found that the CuNWs were actually formed by self-assembling of $\mathrm{Cu}$ nanoparticles along the [110] direction. The transparent electrodes fabricated using the CuNWs achieved a high transparency of $76 \%$ at $31 \pm 5 \Omega / \square$.
\end{abstract}

Key words: $\mathrm{Cu}$ nanowires; growth mechanism; self-assembling; transparent electrodes

\section{Introduction}

Transparent electrodes (TEs) are widely used in various optoelectronic devices, including touch screens, light-emitting diodes, flat-panel displays, and photovoltaic cells. ${ }^{1-3}$ Most of the TEs are made by indium tin oxide (ITO) due to its high transmittance and low resistivity. However, ITO actually is an expensive material as indium is a rare element. In addition, ITO thin film is brittle thus not suitable for flexible devices. ${ }^{4,5}$ The low-cost alternatives such as carbon nanotubes (CNTs), ${ }^{6}$ graphene, ${ }^{7}$ and metal nanowires ${ }^{8}$ have attracted considerable attention. Carbon nanotubes (CNTs) films have been extensively explored, but they have much poorer conductivity compared to ITO at the same transmittance. ${ }^{9}$ Although graphene holds great promise because of its exceptionally high charge mobility and excellent transparency, its sheet resistances (100-1000 $\Omega / \square$ at 80-90\% transmittance) still could not compete with ITO. ${ }^{10}$ Comparatively, thin films of metal nanowires are the most promising alternative because of their high conductivity and optical transmittance. Thin films of silver nanowires (AgNWs) have a transmittance and sheet resistance similar to ITO, ${ }^{11,12}$ but silver is too expensive to be used in a large quantity.

Copper costs much less than silver or indium, and its electrical conductivity is close to silver. ${ }^{13,14}$ Copper nanowires films coated via a solution method have therefore emerged as a perfect alternative to AgNWs or ITO for fabricating low-cost transparent electrodes. ${ }^{15}$ Herein, we reported a facile synthesis of long single-crystalline $\mathrm{Cu}$ nanowires $(\mathrm{CuNWs})$ with excellent dispersibility in ink. We also intuitively observed the self-assembling growth of $\mathrm{Cu}$ nanowires. This self-assembling growth mechanism is quite different with the common growth mechanism in literature where a seeding growth mechanism was normally proposed. It was normally found that the CuNWs were grown from spherical copper seeds,${ }^{16}$ or tapered $\mathrm{Cu}$ nanocrystals. ${ }^{17}$ In addition, Hsing-Yu Tuan observed copper atoms deposition and crystallization on the $\mathrm{Cu}$ nanoparticles to form CuNWs. ${ }^{18}$ However, we observed the $\mathrm{Cu}$ nanocrystals 
self-assembled to form the CuNWs, which is unique among previous observations.

\section{Experimental}

\subsection{Chemicals}

Copper $(\square)$ chloride dihydrate $\left(\mathrm{CuCl}_{2} \cdot 2 \mathrm{H}_{2} \mathrm{O}, \mathrm{AR}\right.$, Aladdin), Nickel( $\left.\square\right)$ acetylacetone (Ni(acac) $)_{2}, 95 \%$, Aladdin), Oleylamine (80-90\%, Aladdin), ethanol (AR, SCR), and hexane (AR, SCR) were all used as received.

\subsection{Synthesis of $\mathrm{Cu}$ nanowires}

CuNWs were synthesized by reducing $\mathrm{CuCl}_{2} \cdot 2 \mathrm{H}_{2} \mathrm{O}$ with oleylamine and $\mathrm{Ni}(\mathrm{acac})_{2}$ as catalyzer, following the approach developed by Guo et al. with some modification. ${ }^{19} \mathrm{In}$ brief, $200 \mathrm{mg} \mathrm{CuCl}{ }_{2} \cdot 2 \mathrm{H}_{2} \mathrm{O}$ and $200 \mathrm{mg} \mathrm{Ni}(\mathrm{acac})_{2}$ were mixed with different volumes $(10,20$, and $30 \mathrm{~mL})$ of oleylamine in a $50 \mathrm{~mL}$ three-necked flask and kept under a flow of common purity Nitrogen at $80 \square$ for 40 min with strong magnetic stirring. After fully dissolution, turn off the magnetic stirring. Then, the solution was heated up to $160 \square$ and kept at this temperature for $4 \mathrm{~h}$, and then cooled to room temperature. The prepared $\mathrm{Cu}$ nanowires were washed by adding hexane and acetone (1:3 in volume) in the reaction mixture with sonication for $2 \mathrm{~min}$, followed by centrifugation at $7000 \mathrm{rpm}$ for $5 \mathrm{~min}$. The washing process was repeated three times. Finally, the $\mathrm{Cu}$ nanowires were kept in hexane.

\subsection{Fabrication of transparent conductive films}

Thin films were prepared by spin-coating method. The glass were degreased with detergent, rinsed with deionized water and ultrasonically cleaned in ethanol for $20 \mathrm{~min}$. Typically, $\mathrm{Cu}$ nanowires were dispersed in hexane by bath sonication for $10 \mathrm{~min}$, and then the CuNWs ink was spin-coated on the cleaned glass at $2000 \mathrm{rpm}$ for $20 \mathrm{~s}$, which repeated five times, and then the films was dried at room temperature after $10 \mathrm{~min}$.

\subsection{Characterization}

The $\mathrm{Cu}$ nanowires and electrodes were characterized by X-ray diffraction measurements (Panalytical X'Pert Pro X-ray power diffractometer using $\mathrm{Cu}-\mathrm{Ka}$ radiation), scanning electron microscope (JSM-5610LV), transmission electron microscope (JEM-2100F STEM/EDS) and optical microscope (OLYMPUS). The transmittance was test on a PerkinElmer UV/VIS/NIR Spectrometer. The sheet resistance was measured using a four-point probe resistivity meter (SDY-6).

\section{Results and discussion}

Our synthesis strategy relies on self-catalytic growth of $\mathrm{Cu}$ nanowires in Oleylamine solution. Upon addition of the precursor, $\mathrm{CuCl}_{2} \cdot 2 \mathrm{H}_{2} \mathrm{O}$, long nanowires were prepared through a $\mathrm{Ni}(\mathrm{acac})_{2}$ catalytic process. Fig. 1a shows the scanning electron microscopy (SEM) images of the $\mathrm{Cu}$ nanowires made by $20 \mathrm{~mL}$ oleylamine. No large bundles were observed, and the nanowires were well dispersed. It can be seen that these CuNWs length is up to $25 \mu \mathrm{m}$. Fig. $1 \mathrm{~b}$ shows the high magnification SEM image of the CuNWs. The average diameter of the nanowires was $\sim 50 \mathrm{~nm}$. The nanowires were found to be single-crystalline and the growth direction of these wires is along [110] as shown in the selected area electron diffraction (SAED) pattern (Fig. 1c).The X-ray diffraction (XRD) pattern in Fig. 1d shows three peaks at $2 \theta=43.5,50.7$, and $74.4^{\circ}$, which correspond to the (111), (200) and (220) planes of a FCC Cu (JCPDS \#03-1018) respectively. No other peaks were observed, confirming the pure copper nanowires were formed. 
Fig.1 (a) and (b) SEM images of $\mathrm{Cu}$ nanowires. (c) SAED pattern of the $\mathrm{Cu}$ nanowires shown in the inset. (d) XRD pattern of $\mathrm{Cu}$ nanowires.

The structure of the nanowires was further studied by the high-resolution TEM. On the basis of SAED and XRD analysis, these nanowires were single-crystalline and grew along the [110] direction. Such, the side faces of nanowires should be the (200), (111) planes which was confirmed by the corresponding high-resolution TEM images (Figure. 2a,c) taken from the middle and end portions of two different $\mathrm{Cu}$ nanowires. The Figure. (2b.c) clearly showed fringes corresponding to the (111), (200) planes along the axis of the nanowires.

Fig.2 (a,c) TEM images taken from two different Cu nanowires; (b,d) HRTEM images of the boxed areas in (a) and (c), respectively.

In order to study the growth mechanism, we stopped the CuNWs reaction at different times and examined the product with electron microscopy. At the initial stage ( $\mathrm{t}=30 \mathrm{~min}$ ), the SEM image (Fig. 3a) showed the $\mathrm{Cu}$ nanoparticles whose diameter gradually changed from $40 \mathrm{~nm}$ to $180 \mathrm{~nm}$ were formed, which exhibited the development of nanowire embryos from the nanoparticles. After the reaction had proceeded to $t=1 \mathrm{~h}$ (Fig. $3 \mathrm{~b}, 3 \mathrm{c}$ ), we observed that the nanoparticles (diameter of $100 \mathrm{~nm}$ ) began to self-assemble into a line. As the reaction was continued to $2 \mathrm{~h}$ (Fig. 3d), the assembled $\mathrm{Cu}$ nanoparticles grew into nanowires. Then after $2.5 \mathrm{~h}$ of reaction (Fig. $3 \mathrm{e}$ ), it was found that the surface of $\mathrm{Cu}$ nanowires became smooth although the $\mathrm{Cu}$ nanowires were incomplete fusion. These results indicate that the $\mathrm{Cu}$ nanowires were originated from nanocrystals, through a one to one self-assembling between the initial seeds. This self-assembling growth mechanism is unique among the preciously observed seeds-growth mechanism where $\mathrm{Cu}$ nanoparticles were formed and acted as seeds that started to grow into nanowires along a specific crystal orientation..$^{22,23}$

Fig.3 (a-d) SEM images of Cu nanowires synthesized ed at reaction times of (a) 0.5, (b,c) 1, (d) 2, (e) $2.5 \mathrm{~h}$.

Why the nanocrystals would like to self-assemble to form nanowires? When the reaction is under thermodynamic control, $\mathrm{Cu}$ nanocrystals attempt to minimize the total surface energy. ${ }^{24}$ For an FCC structure, the energetic sequence of $\gamma(111)<\gamma(100)<\gamma(110)$. This implies that $\mathrm{Cu}$ nanocrystals should maximize the exposed (111) facets. The oleylamine is an effective capping agent for $\mathrm{Cu}$, with a good selectivity toward the (200) facets. Besides, The $\mathrm{Cl}^{-}$ions forming $\mathrm{CuCl}_{2}$ precursor is attached onto the (200) facets and leads to a strongly anisotropic growth. ${ }^{25}$ Therefore, the $\mathrm{Cu}$ nanocrystals would be inclined to be connected by the active (110) facets, leading to preferential growth of the nanowires along the [110] direction. This is well consistent with the SAED and TEM results.

It is important to mention that with the absence of $\mathrm{Ni}(\mathrm{acac})_{2}$, we did not observe any particles or nanowires even after $10 \mathrm{~h}$ reaction. Because $\mathrm{Cu}^{2+}$ ions were strongly coordinated by $\mathrm{Cl}^{-}$, which prevents $\mathrm{Cu}^{2+}$ from being reduced. This suggests the catalytic role of $\mathrm{Ni}^{2+}$ ions during the $\mathrm{Cu}$ nanowires formation. The $\mathrm{Ni}^{2+}$ ions were reduced by oleylamine to form $\mathrm{Ni}(0)$, then the oxidation of $\mathrm{Ni}(0)$ back to $\mathrm{Ni}^{2+}$ ions during the galvanic replacement reaction with $\mathrm{Cu}^{2+}$ ions.

On the basis of the above observations and analysis, the formation mechanism can be proposed (Fig. 4). Firstly, $\mathrm{Ni}^{2+}$ ions were reduced to $\mathrm{Ni}(0)$ atoms in oleylamine solution. Secondly, Upon the addition of $\mathrm{Cu}(\mathrm{Cl})_{2}$, the precursor rapidly involves into the galvanic replacement reaction with $\mathrm{Ni}(0)$. Then, the $\mathrm{Cu}$ nanocrystalline seeds start to form. Subsequently, the nanoparticles began to assemble into a line and 
connect to form nanowires by Ostwald ripening along the [110] direction, as their side (200) facts were stabilized by oleylamine and $\mathrm{Cl}^{-}$. Thus a long $\mathrm{Cu}$ nanowire is formed.

Fig.4 Schematic of the formation mechanism of CuNWs.

Surfactants typically play a crucial role in controlling the size and shape of chemically synthesized nanoparticles. So we can control the diameter of CuNWs by adjusting oleylamine volume. Fig. 5a-f shows the SEM images of the CuNWs in various volumes of oleylamine $(10,20,30 \mathrm{~mL}$, respectively). At low oleylamine concentration, the average diameter of CuNWs (Fig. 5a, b) is $20 \mathrm{~nm}$ and the length is $15-40$ $\mu \mathrm{m}$. By increasing the oleylamine volume to $20 \mathrm{~mL}$, the average diameter of CuNWs (Fig. 5c, d) increases to $50 \mathrm{~nm}$, and the length is $15-25 \mu \mathrm{m}$. Further increasing the oleylamine volume to $30 \mathrm{~mL}$, the CuNWs (Fig. 5e,f) are wide $(120 \mathrm{~nm})$, and short $(4-16 \mu \mathrm{m})$. It can be concluded that the aspect ratio of the CuNWs decreases with the increase in the concentration of oleylamine. It is believed that the decrease in the oleylamine amount resulted in the formation of small size of the assembled $\mathrm{Cu}$ nanoparticles. ${ }^{26}$ Therefore, with the decrease in oleylamine amount, thinner and longer $\mathrm{Cu}$ nanowires were formed by the self-assembling of smaller nanoparticles (Fig. S1†). The study concludes that the diameter of $\mathrm{Cu}$ nanoparticles formed at the initial stage restricts the final diameter and length of CuNWs.

Figure 5. SEM image of Cu nanowires prepared with different volumes of oleylamine: (a, b) $10 \mathrm{ml}$; (c, d) $20 \mathrm{ml}$; (e, f) $30 \mathrm{ml}$.

Two types of the prepared CuNWs, long $(15-25 \mu \mathrm{m})$, thin $(<60 \mathrm{~nm})$ CuNWs and the longer $(15-40$ $\mu \mathrm{m})$, thinner $(20 \mathrm{~nm})$ CuNWs were made into well-dispersed CuNWs ink separately and then coated as transparent electrodes on glass. The UV-Vis spectra (Fig. 6a) show that the transmittance of the two films.made by the different CuNWs is similar $(\mathrm{T}=76 \%$ ). However, the longer and thinner CuNWs exhibited a lower sheet resistance of $31 \pm 5 \Omega / \square$ compared to the other one (43 $\pm 4 \Omega / \square$ ), which is comparable to the CuNWs TEs obtained by Wiley and co-workers with a sheet resistance $61 \Omega / \square$ at $67 \%$ transmittance. ${ }^{14}$ This difference in conductivity is accounted for the difference in nanowire length and diameter. The CuNWs films with low aspect ratio leave more open space than films of the CuNWs with high aspect ratio (Fig. 6b, c). Therefore, films of the CuNWs with high aspect ratio exhibited a lower sheet resistance at the same transmittance.

Fig. 6 (a) UV-Vis transmittance spectra for two different $\mathrm{Cu}$ nanowires electrodes, and the optical microscope images of (b) higher aspect ratio $\mathrm{Cu}$ nanowires ( $\mathrm{T}=76 \%$, $\mathrm{Rs}=31 \pm 5 \Omega / \square$ ) and (c) lower aspect ratio $\mathrm{Cu}$ nanowires $(\mathrm{T}=76 \%, \mathrm{Rs}=43 \pm 4 \Omega / \square)$.

\section{Conclusions}

In conclusion, we have shown that self-catalytic and self-assembling growth mechanism of the CuNWs, with the active Ni2+, Cl- ions and oleylamine as a critical role in synthesis of stable CuNWs. 15-40 $\mu \mathrm{m}$ long single crystalline CuNWs were synthesized and made into ink for preparation of transparent electrodes, achieving $31 \pm 5 \Omega / \square$ at $76 \%$ transparency. With the superior properties and low cost, CuNWs are believed to be the most promising alternative of ITO.

\section{Reference}


[1] Lee JY, Connor ST, Cui Y, et al. Semitransparent organic photovoltaic cells with laminated top electrode[J]. Nano lett., 2010, 10(4): 1276.

[2] Gomez De Arco L, Zhang Y, Schlenker CW, et al. Continuous, highly flexible and transparent graphene films by chemical vapor deposition for organic photovoltaics[J]. ACS Nano., 2010, 4(5): 2865.

[3] Borchert JW, Stewart IE, Ye S, et al. Effects of length dispersity and film fabrication on the sheet resistance of copper nanowire transparent conductors[J]. Nanoscale., 2015, 7(34): 14496.

[4] Leterrier Y, Medico L, Demarco F, et al. Mechanical integrity of transparent conductive oxide films for flexible polymer-based display[J]. Thin Solid Films., 2004, 460(1-2): 156.

[5] Ravi Kumar DV, Woo K, Moon J. Promising wet chemical strategies to synthesize Cu nanowires for emerging electronic applications[J]. Nanoscale., 2015, 7(41): 17195.

[6] Wang RR, Sun J, Gao LA, et al. Base and acid treatment of SWCNT-RNA transparent conductive films [J]. ACS Nano., 2010, 7(41): 17195.

[7] Wang RR, Sun J, Gao LA, et al. Effective post treatment for preparing highly conductive carbon nanotube reduced graphite oxide hybrid films[J]. Nanoscale., 2011, 3(3): 904.

[8] Hu L, Kim HS, Lee JY, et al. Scalable coating and properties of transparent, flexible, silver nanowire electrodes[J]. ACS Nano., 2010. 4(5): 2955.

[9] Van de Lagemaat J, Barnes TM, Rumbles G, et al. Organic solar cells with carbon nanotubes replacing $\mathrm{Zn}_{2} \mathrm{O}_{3}$ : Sn as the transparent electrode[J]. App. Phys. Lett., 2006. 88(23): 233503.

[10] Kim UJ, Lee IH, Bae JJ, et al. Graphene\ Carbon nanotube hybrid-based transparent 2D optical array[J]. Adv. Mater. 2011, 23(33): 3809.

[11] De S, Higgins TM, Lyons PE, et al. Silver nanowire network as flexible, transparent, conducting films: extremely high DC to optical conductivity ratios[J]. ACS Nano., 2009, 3(7): 1767.

[12] Zhang DQ, Wang RR, Wen M C. et al. Synthesis of ultralong copper nanowires for high-performance transparent electrode[J]. J. Am. Chem. Soc., 2012, 134(35): 14283.

[13] Rathmell AR, Wiley BJ. The synthesis and coating of long, thin copper nanowires to make flexible, transparent conducting films on plastic substrates[J]. Adv. Chem. Soc. 2011, 23(41): 4798.

[14] Ye S, Stewart IE, Chen ZF. et al. How copper nanowires grow and how to control their properties[J]. Acc. Chem. Res., 2016, 49(3): 442.

[15] He C, Liu G, Zhang WX. et al. Tuning the structure and electron transport properties of ultra thin $\mathrm{Cu}$ nanowire by size and bending stress using DFT and FTB methods[J]. RSC. Adv., 2015, 5(29): 22463.

[16] Rathmell AR, Bergin SM, Hua YL. et al. The growth mechanism of copper nanowires and their properties in flexible, transparent conducting films[J]. Adv. Mater., 2010, 22(32): 3558.

[17] Jin SM, He GN, Zhang H. et al. Shape-controlled synthesis of copper nanowires and their properties in flexible, transparent conducting films[J]. Angew. Chem. Int. Ed., 2011, 50(45): 10560. 
[18] Yang HJ, He SY, Tuan HY. Self-seeded growth of Five-Fold twinned copper nanowires: Mechanistic study, characterization, and SERS applications[J]. Langmuir., 2014, 30(2): 602.

[19] Guo HZ, LinN, Chen YZ. et al. Copper nanowires as fully transparent conductive electrodes. [J]. Sci. Rep. 2013,3: 2323.

[20] Meng F, Jin S. The solution growth of copper nanowires and nanotubes is driven by screw dislocations[J]. Nano. Lett., 2012, 12(1): 234.

[21] Ye S, Rathmell AR, Stewart IE. et al. Rapid synthesis of high aspect ratio copper nanowires for high-performance transparent conducting films[J]. Chem. Commun., 2014, 50(20): 2562.

[22] Xia YN, Xiong YJ, Lim B. et al. Shape-controlled synthesis of metal nanocrystals: simple chemistry meets complex physics[J]. Angew. Chem. Int. Ed., 2009, 48(1): 60.

[23] Nam VB,Lee D. Copper nanowires and their applications for flexible, transparent conducting films: A review[J]. Nanomater., 2016, 6(3): 47.

[24] Nandwana V, Elkins KE, Poudyal N, et al. Size and shape control of monodiperse FePt Nanoparticles[J]. J.Phys. Chem. C., 2007, 111(11): 4185. 

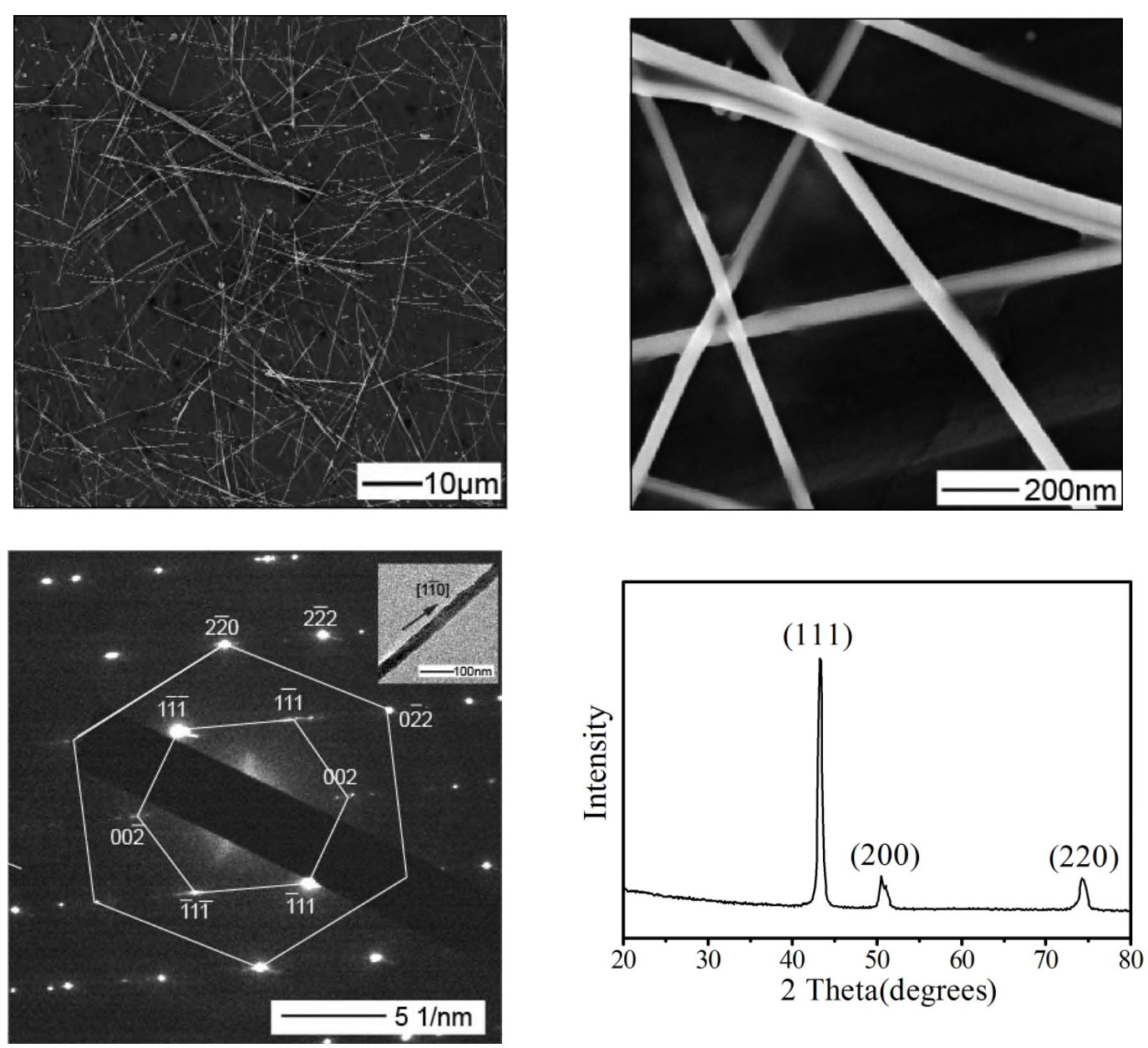

Figure 1. (a) and (b) SEM images of $\mathrm{Cu}$ nanowires. (c) SAED pattern of the $\mathrm{Cu}$ nanowires shown in the inset. (d) XRD pattern of $\mathrm{Cu}$ nanowires. 
Figure 2. (a,c) TEM images taken from two different Cu nanowires; $(b, d)$ HRTEM images of the boxed areas in (a) and (c), respectively.
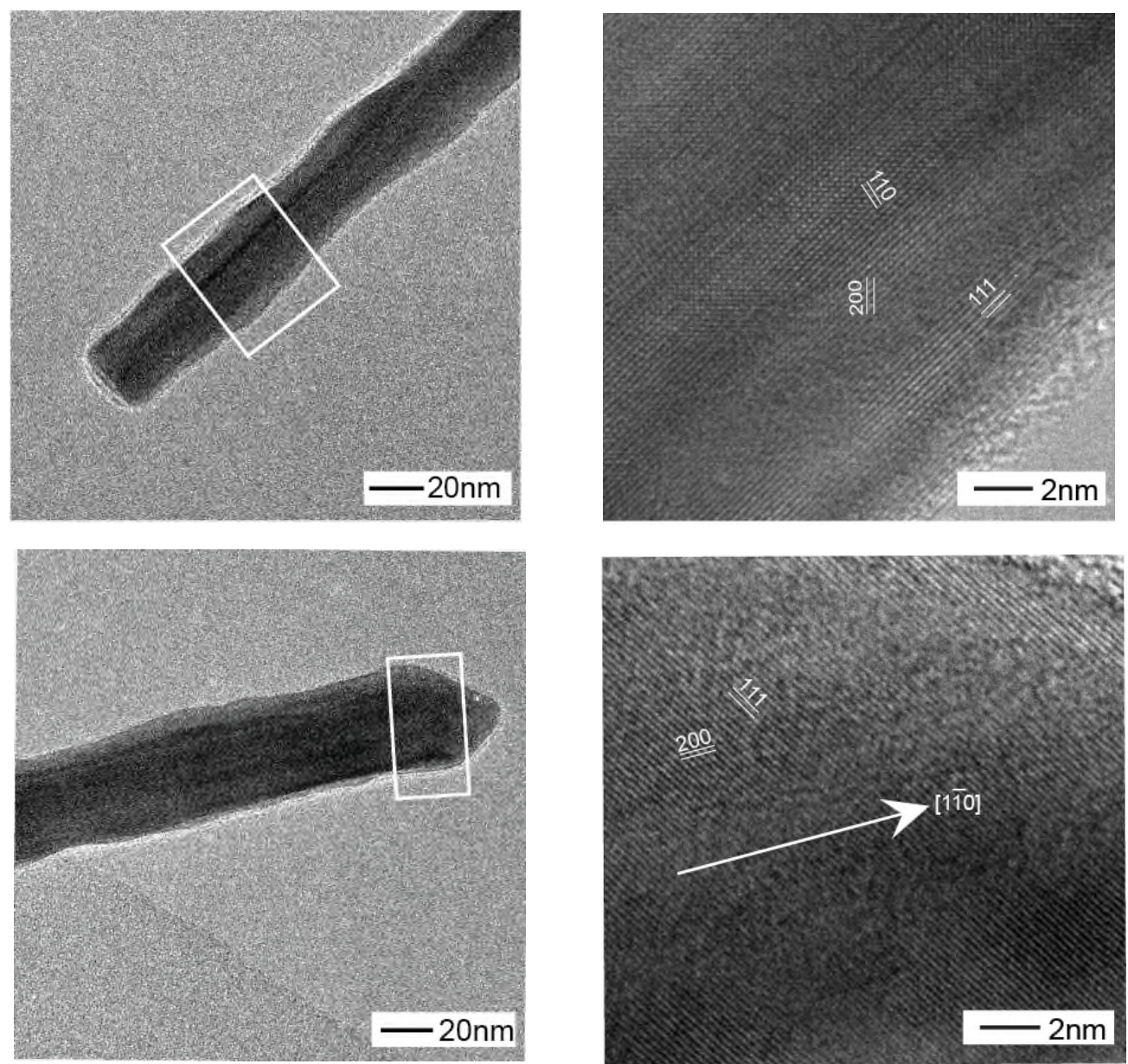
Figure 3. (a-d) SEM images of Cu nanowires synthesized ed at reaction times of (a) 0.5 , (b,c) 1, (d) 2, (e) 2.5 h.
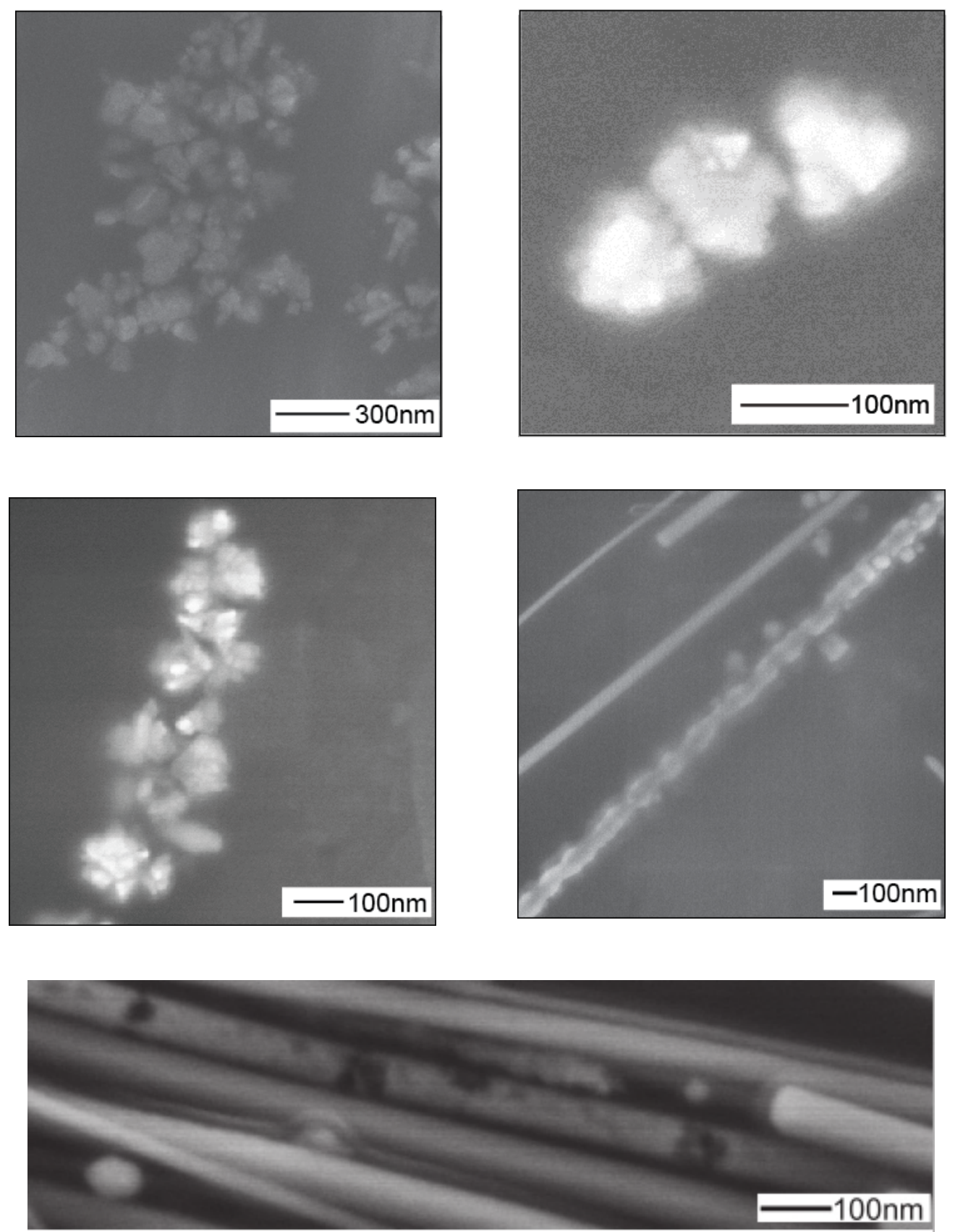
Figure 4. Schematic of the formation mechanism of CuNWs.

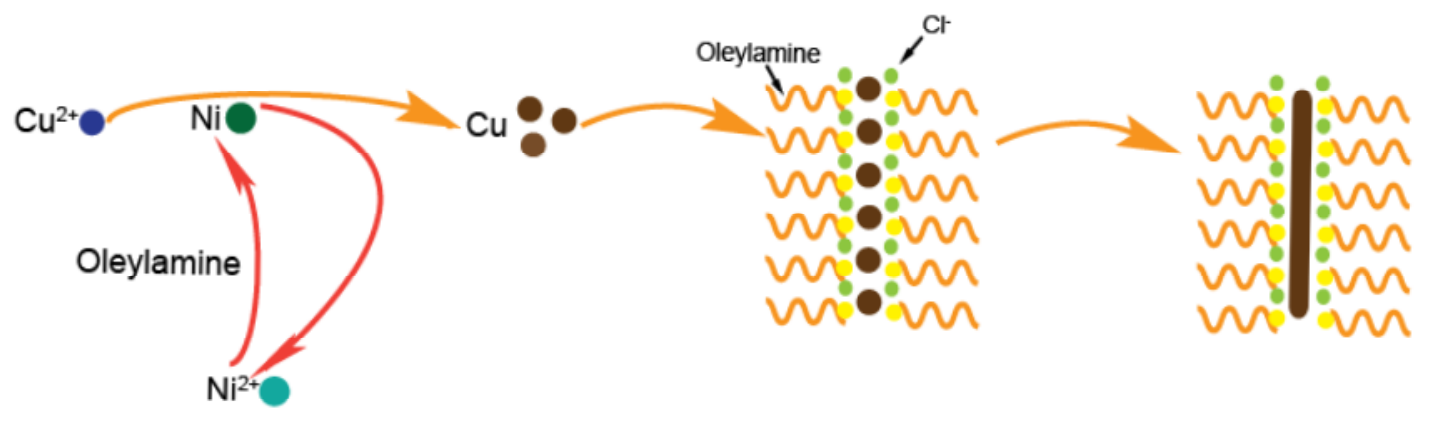

Figure 5. (a) the optical microscope image of $\mathrm{Cu}$ nanowires electrodes with $76.43 \%$ transmittance. (b) UV-Vis-NIR wavelength transmission spectra for $\mathrm{Cu}$ nanowire electrode (sheet resistance $\sim 43.28 \Omega / \square$ ) .
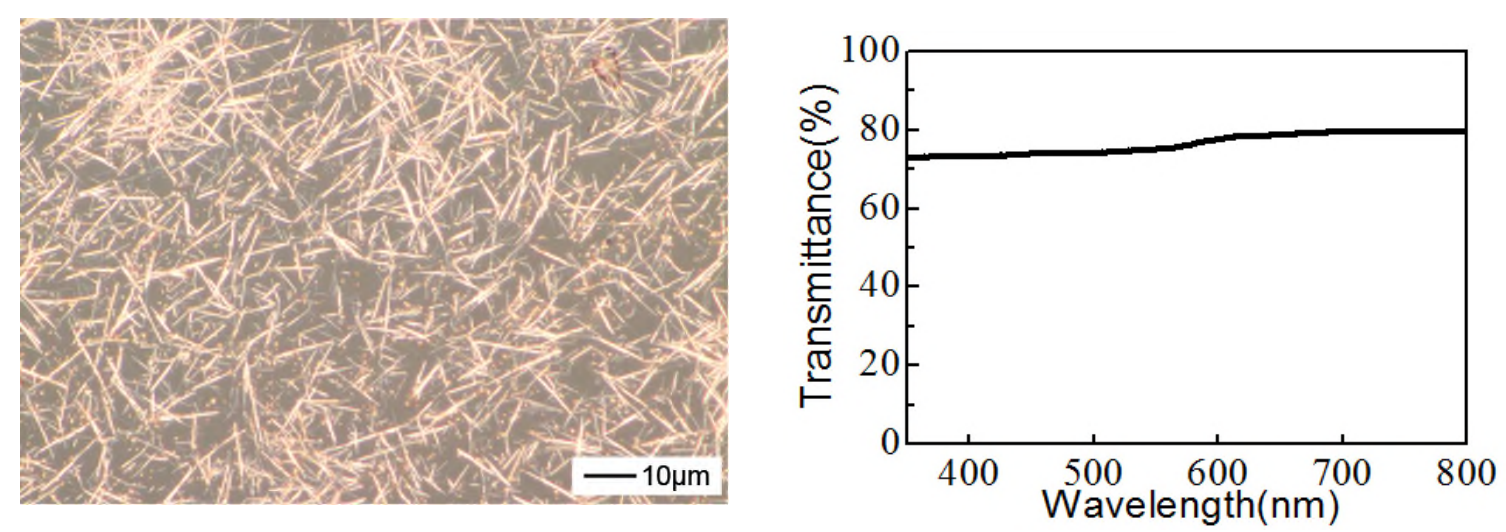
2019-02-18

\section{The self-assembling growth of copper nanowires for transparent electrodes}

Wu, Junqing

Springer

Junqing Wu, Le Guo, Min Wen, et al., (2019) The self-assembling growth of copper nanowires for transparent electrodes. Journal of Wuhan University of Technology (Materials Science pÿEdition), Volume 34, Issue 1, February 2019, pp. 145149

https://doi.org/10.1007/s11595-019-2028-8

Downloaded from Cranfield Library Services E-Repository 